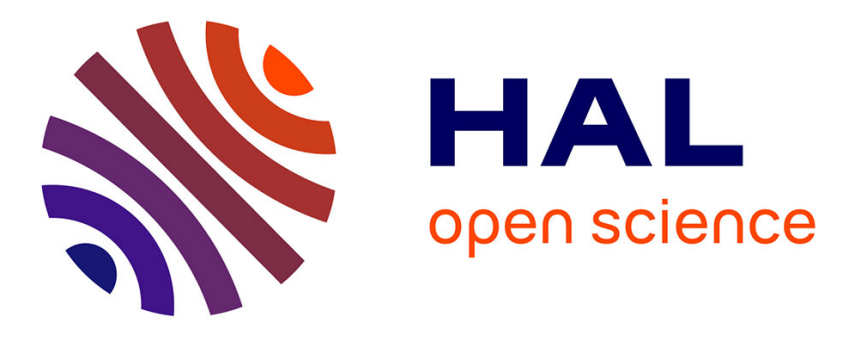

\title{
Experimental Measurement of Human Oocyte Mechanical Properties on a Micro and Nanoforce Sensing Platform Based on Magnetic Springs.
}

Joël Abadie, Christophe Roux, Emmanuel Piat, C. Filiatre, Caroline Amiot

\section{- To cite this version:}

Joël Abadie, Christophe Roux, Emmanuel Piat, C. Filiatre, Caroline Amiot. Experimental Measurement of Human Oocyte Mechanical Properties on a Micro and Nanoforce Sensing Platform Based on Magnetic Springs.. Sensors and Actuators B: Chemical, 2014, 190, pp.429-438. 10.1016/j.snb.2013.08.042 . hal-01002533

\section{HAL Id: hal-01002533 https://hal.science/hal-01002533}

Submitted on 6 Jun 2014

HAL is a multi-disciplinary open access archive for the deposit and dissemination of scientific research documents, whether they are published or not. The documents may come from teaching and research institutions in France or abroad, or from public or private research centers.
L'archive ouverte pluridisciplinaire HAL, est destinée au dépôt et à la diffusion de documents scientifiques de niveau recherche, publiés ou non, émanant des établissements d'enseignement et de recherche français ou étrangers, des laboratoires publics ou privés. 


\title{
Experimental Measurement of Human Oocyte Mechanical Properties on a Micro and Nanoforce Sensing Platform Based on Magnetic Springs
}

\author{
J. Abadie ${ }^{\mathrm{a}, *}$, C. Roux ${ }^{\mathrm{b}}$, E. Piat ${ }^{\mathrm{a}}$, C. Filiatre ${ }^{\mathrm{c}}$, C. Amiot $^{\mathrm{b}}$ \\ ${ }^{a}$ FEMTO-ST Institute, UMR CNRS 6174, UFC - ENSMM - UTBM, Besançon, France \\ ${ }^{b}$ Génétique, Histologie et Biologie du Développement et de la Reproduction, INSERM UMR1098, CIC-BT506, CHRU de \\ Besançon, France \\ ${ }^{c}$ UTINAM Institute, UMR CNRS 6213, Université de Franche-Comté, Besançon, France
}

\begin{abstract}
This article presents a new micro and nanoforce sensor used to perform a mechanical characterisation of human oocytes. This device is based on the use of low-stiffness magnetic springs. The oocytes to be characterised are placed on a force-sensitive platform. A manipulator equipped with a standard micropipette is used to mechanically compress the oocyte. Some complete "force-compression length" curves associated with mechanical load-unload cycles are given. These curves show the linear, the non-linear and also the plastic mechanical behaviour of the oocytes. These characterisations must be considered as a preliminary result which illustrates that the mechanical variability and the mechanical evolution of human oocytes during their maturation process can be observed with a force sensor based on magnetic springs.
\end{abstract}

Keywords: micro and nanoforce sensor, oocyte mechanical characterisation, magnetic springs.

*Corresponding author.

Email address: jabadie@femto-st.fr (J. Abadie)

Preprint submitted to Elsevier

September 3, 2013 


\section{Introduction}

As mentioned at the beginning of [1], palpation remains an important approach in the medical diagnosis of organ illness, and the mechanical translation of this physician's approach leads to the cited fact that "stiffness is an important parameter in determining the physical properties of living tissue" and thus to identify healthy or diseased parts of tissue during the patient diagnosis. As recently suggested, this approach should be generalized to oocyte selection in assisted reproductive technology (ART) if the physician could have the ability to feel the stiffness of the candidate oocytes. Furthermore, Woodruff emitted the hypothesis that the physical properties of the follicle environment, in vivo (in normal ovary or in ovarian disorders) or in vitro (in 3D matrices culture), modulates the follicles responsiveness to paracrine factors and hormones [2]. The study of such biomechanical signalling, particularly for in vitro culture, will require the development of new tools and new approaches. With the important development of micro-mechanical systems, the measurement of very low stiffness on biological tissues becomes possible $[3,4]$ and considerable biomedical attention is focused on the mechanical properties of living tissues at the single cell level [1]. Stiffness measurement at this scale requires very sensitive micro and nanoforce sensors. Many sensors have already been developed and present a force resolution compatible with the needs of ART [5, 6]. Authors have also proposed different rheology techniques used to characterise the internal or global mechanical parameters of biological cells. These techniques are based on optical techniques associated with a modelling of the cell $[7,8,9]$ or on the use of glass elastic microstructures [10]. Mechanical properties of the cytoplasm of oocytes have also been studied thanks to fluorescent microbeads microinjected inside starfish oocytes [11]. This multiple-particles tracking approach has shown that the maturation process in starfish oocytes is accompanied by a change in the mechanical response of the cytoplasm. Despite its efficiency, such a technique will probably not be approved by the french Biomedecine Agency for human oocytes fertilisation using an ICSI ${ }^{1}$ protocol. This agency requires that all new manipulation techniques or tools added to the ICSI routine protocol on human oocytes, have no proven deleterious effects. As a result, none of these cited techniques have been applied to human assisted reproductive technology.

The oocyte selection plays a critical role in the initial embryo development and therefore in ART results. The attempts concern in vitro fertilization (IVF) or intra cytoplasmic sperm injection (ICSI). Oocyte quality depends on its nuclear and cytoplasmic maturation stage. The quality estimation during ICSI procedure is based on morphological criterion after the removal of the cumulus-corona cells. A mature human oocyte has a single polar body in the perivitelline space (PVS), a normal aspect of the cytoplasm and of the zona pellucida (ZP) $[12,13]$. Furthermore, observation of oocyte birefringent structures with polarized light microscopy is useful for observing the meiotic nuclear maturation status by displaying the meiotic spindle (MS) and analyzing the highly-ordered inner layers of the ZP $[14,15]$. However morphology is biologist-dependant and often fails to predict fertilizing ability and developmental competence.

During oocyte growth and maturation, both cytoplasmic modifications, and changes in the ZP structures and thickness are responsible for the variation of the oocyte stiffness. After fecundation occurs, the modification of the zona pellucida, called the zona pellucida hardening (consecutive to the release of the cortical granules from the cytoplasm of the oocyte in the PVS), participates in the change of the stiffness. Furthermore, different culture conditions which alter the ZP structure may also change the stiffness.

The oocyte characteristics have been investigated by means of mechanical measurements using micropipettes, different indenter tips, or systems consisting of pressing oocytes on a "wall" or a holding micropipette. These studies are focused on mammalian [16, 1, 17, 18, 19] or human oocytes $[20,21]$. These previous works show that immature

\footnotetext{
${ }^{1}$ Intracytoplasmic Sperm Injection
} 
and mature oocytes or young and old, are mechanically different $[21,22]$. Furthermore, characterisation of the cellular forces applied to the oocyte during microinjection may assess oocyte stiffness at this specific ART event. To perform these tasks, new instrumentation compatible with ART constraints and physicians' habits is necessary. With their low stiffness and their high ranges of force measurement, force sensors based on magnetic springs [23, 24, 25] could be a potential solution to design such instrumentation.

This article describes a new oocyte characterisation device based on the past experience gathered on a floating platform using a magnetic spring dynamometer [26]. Some preliminary experimental results of mechanical characterisations done with mature and immature human oocytes are given. Rather than calculating the stiffness or Young's modulus of human oocytes, the complete force-compression length curves associated with mechanical load-unload cycles are given. These curves bring new and more complete information. They show the non-linear and also the plastic mechanical behaviour of human oocytes. These characterisations have to be considered as a preliminary result which illustrates that the mechanical variability and the mechanical evolution of human oocytes during their maturation process can be observed with a force sensor based on magnetic springs. This device has been developed at the FEMTO-ST institute by the SPECIMeN ${ }^{2}$ group of the $\mathrm{AS}_{2} \mathrm{M}^{3}$ department.

\section{Description of the force sensing platform}

The force sensor based on magnetic springs that has been prepared to characterise oocytes is a prototype that is able to measure a vertical torque and micro and nanoforces in two perpendicular horizontal directions of space [26]. The oocytes that have to be characterized are placed at the centre of a floating

\footnotetext{
${ }^{2}$ Sensing strategies, Perception and Characterisation at Micro and Nanoscales

${ }^{3}$ Automatic Control and Micro-Mechatronic Systems
}

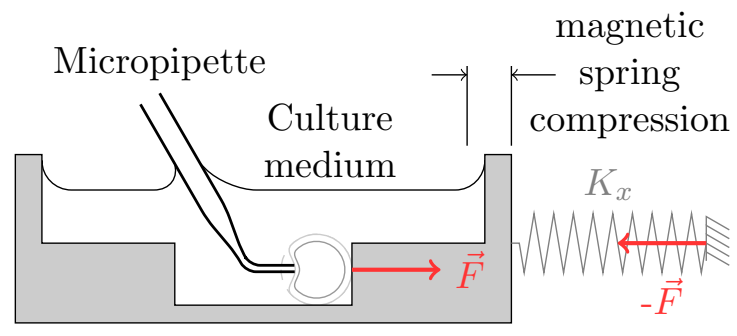

Special Petri dish

Figure 1: Forces applied on the force sensing device when an oocyte is under a compression test.

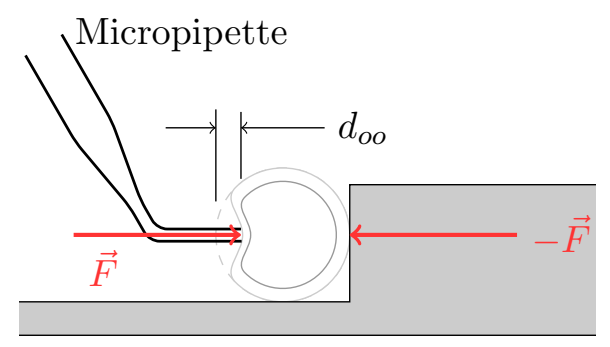

Figure 2: The compressed oocyte with the forces applied on it and the resulting compression length $d_{o o}$.

platform in a special Petri dish filled with a culture medium. An external manipulator ${ }^{4}$ equipped with an ICSI micropipette is used to apply mechanical loads on the oocyte.

\subsection{Simplified description of the force sensing plat- form}

Fig. 1 shows the principle of the force sensing platform used to characterise the mechanical response of the oocyte to a compression cycle. It is composed of a force sensor device that is used to measure the compression force $\vec{F}$ applied to the oocyte (see Fig. 2). The Eppendorf manipulator is used to generate displacements of a micropipette and control the force applied on the oocyte. The oocyte remains in a culture medium during the compression cycle to preserve it from the environment. It is pushed by the moving micropipette against a vertical surface (a machined vertical well with important dimensions com-

\footnotetext{
${ }^{4}$ Eppendorf TransferMan NK2
} 


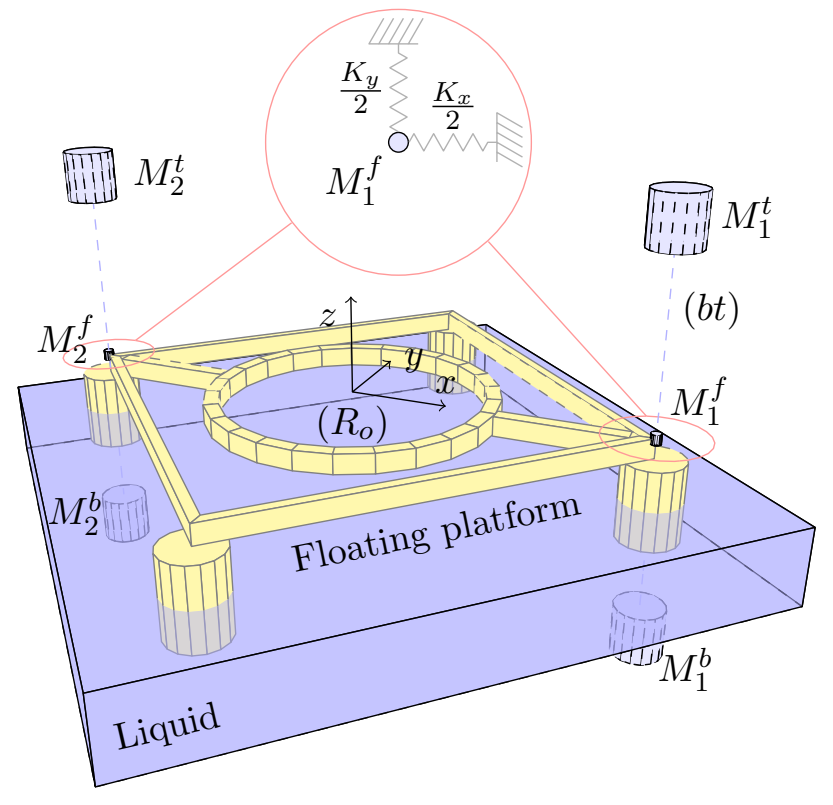

Figure 3: Principle of the force sensing platform.

pared to the diameter of the oocyte). When a force $\vec{F}$ is applied on the well, the magnetic spring $K_{x}$ is compressed (see Fig. 1). Similar to a classical dynamometer, the compression length of the magnetic spring is proportional to the force applied on it, allowing a force calculation when the spring compression length is measured. Finally, the simultaneous measurement of $\vec{F}$ and compression length $d_{o o}$ (see Fig. 2) provides some behaviours of the oocyte under mechanical compression that will be presented in section 4 .

\subsection{Physical principle of the force sensing device}

The force sensing device is mainly composed of a floating platform. This floating platform constitutes the mobile part of a dynamometer on which the force to be measured is applied in the plane $(x O y)$ as shown in Fig. 3 and 4. Several magnetic springs are used to create a link between the platform and the stand of the system, to which a reference frame $\left(R_{o}\right)$ is attached. The fixed top and bottom magnets $M_{1}^{t}$ and $M_{1}^{b}$ shown in Fig. 3 generate a force field centered on the vertical line $(b t)$ going through them. As a result,

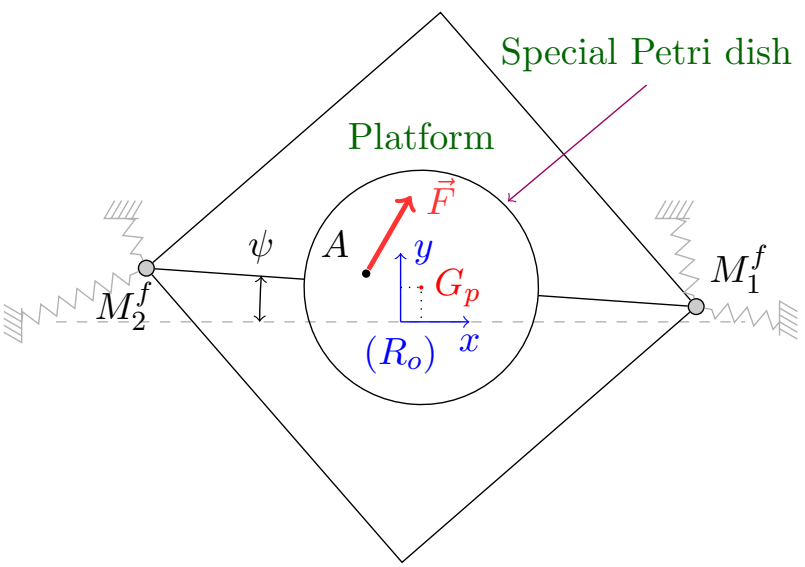

Figure 4: Behaviour of the floating platform when a force $\vec{F}$ is applied on it.

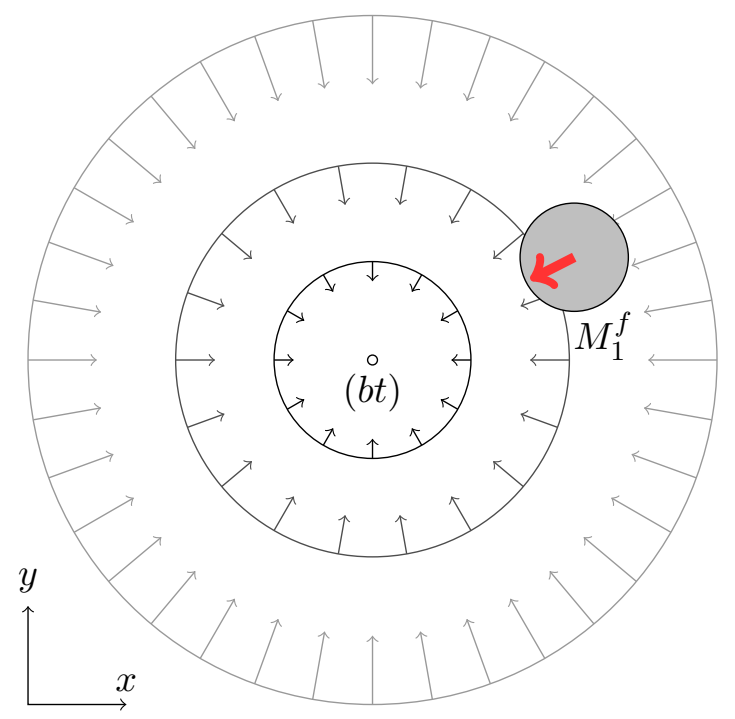

Figure 5: Force field generated perpendicularly to the vertical line $(b t)$ by magnets $M_{1}^{t}$ and $M_{1}^{b}$ and applied to $M_{1}^{f}$ (magnetic spring). 
the little magnet $M_{1}^{f}$, which is fixed on the platform, is constrained into a concentric force field (see Fig. 5) that is modeled by two orthogonal magnetic springs of stiffness $\frac{K_{x}}{2}$ and $\frac{K_{y}}{2}$. The same magnet configuration is reproduced at the other extremity of the platform (magnets $M_{2}^{b}, M_{2}^{t}$ and $M_{2}^{f}$ ) providing a 3DOF dynamometer of stiffness $K_{x}$ and $K_{y}$ along the $x$ and $y$ directions and a stiffness $K_{\psi}$ for the rotation $\psi$ around the $z$ axis. The use of this axis direction is not suitable for microforce measurement because the $z$ displacement of the platform is not measured. When an external force $\vec{F}=\left(F^{x}, F^{y}\right)^{T}$ is applied to the platform at a point $A$, the position of the platform's center of gravity, $G_{p}$, is modified (see Fig. 4). In steady state, according to [26], the relationship between the force and the displacement of the platform is given by Eq.:

$$
\begin{gathered}
\vec{F}=\mathbf{K}_{\mathbf{m}} \cdot \vec{X} \\
\Leftrightarrow\left(\begin{array}{c}
F^{x} \\
F^{y} \\
\mathcal{M}_{F_{/ G_{p}}}
\end{array}\right)=\left(\begin{array}{ccc}
K_{x} & 0 & 0 \\
0 & K_{y} & 0 \\
0 & 0 & K_{\psi}
\end{array}\right)\left(\begin{array}{c}
G_{p}^{x} \\
G_{p}^{y} \\
\sin \psi
\end{array}\right)
\end{gathered}
$$

in which the vector $\vec{X}$ contains the coordinates and orientation of the platform in the reference frame $\left(R_{o}\right)$ attached to the stand. $\mathbf{K}_{\mathbf{m}}$ is the stiffness matrix of the magnetic springs and $\mathcal{M}_{F_{/ G_{p}}}$ is the $z$ component of the momentum of the force $\vec{F}$ around $G_{p}$. Assuming that $M_{1}^{f} M_{2}^{f}=2 \delta$, for a small rotation angle $\psi$, the rotational stiffness of the platform is :

$$
K_{\psi}=\delta^{2} K_{y}
$$

With this dynamometer, all the unattended dry friction forces are eliminated thanks to the liquid and floating principle. The viscous friction forces are canceled in steady states. The liquid menisci on the floats have no detectable effect on the force measurement [26].

\subsection{Estimation of the force applied to the platform}

To determine the force $\vec{F}$ that is applied onto the platform, it is necessary to measure the position of $G_{p}$ in $\left(R_{o}\right)$. Then, the Eq. (1) is used to calculate

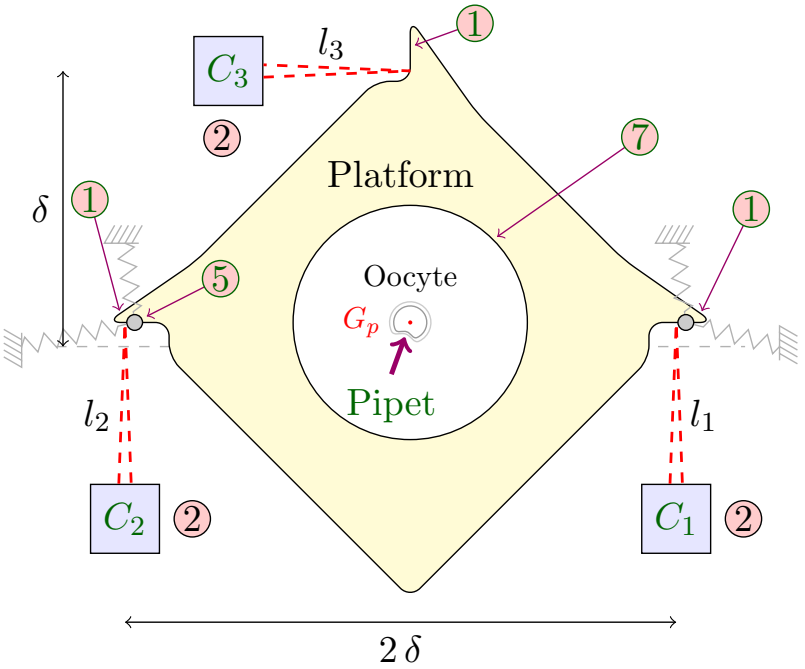

Figure 6: Displacement measurement of the platform with confocal sensors.

the estimation $\hat{\vec{F}}$ of the applied force $\vec{F}$ :

$$
\hat{\vec{F}}=\left(\begin{array}{c}
\hat{F}^{x} \\
\hat{F}^{y}
\end{array}\right)=\left(\begin{array}{cc}
K_{x} & 0 \\
0 & K_{y}
\end{array}\right)\left(\begin{array}{l}
\hat{G}_{p}^{x} \\
\hat{G}_{p}^{y}
\end{array}\right)
$$

To determine the stiffness $K_{x}$ and $K_{y}$ of the magnetic springs, a calibration process is performed (see Section 2.6). One can note that $\mathcal{M}_{F_{/ G_{p}}}$ will produce a rotation $\psi$ of the platform that has no influence on the force estimation.

\subsection{Experimental setup for oocytes characterisation}

High resolution chromatic confocal displacement sensors $^{5}$ are used to measure the position of $G_{p}$ (see Section 2.5). This choice requires the platform to be fitted with three deflectors labeled (1) in Fig. 6 . These are glass deflectors with nanometric roughness. The three confocal sensors (2) are targeted on them. The experimental platform also includes the following parts shown in Fig. 6 and 7:

(3) fixed permanent magnets $M_{1}^{t}, M_{1}^{b}$,

(4) fixed permanent magnets $M_{2}^{t}, M_{2}^{b}$,

${ }^{5}$ Stil confocal sensor, model CL4, $0.1 \mu \mathrm{m}$ resolution 


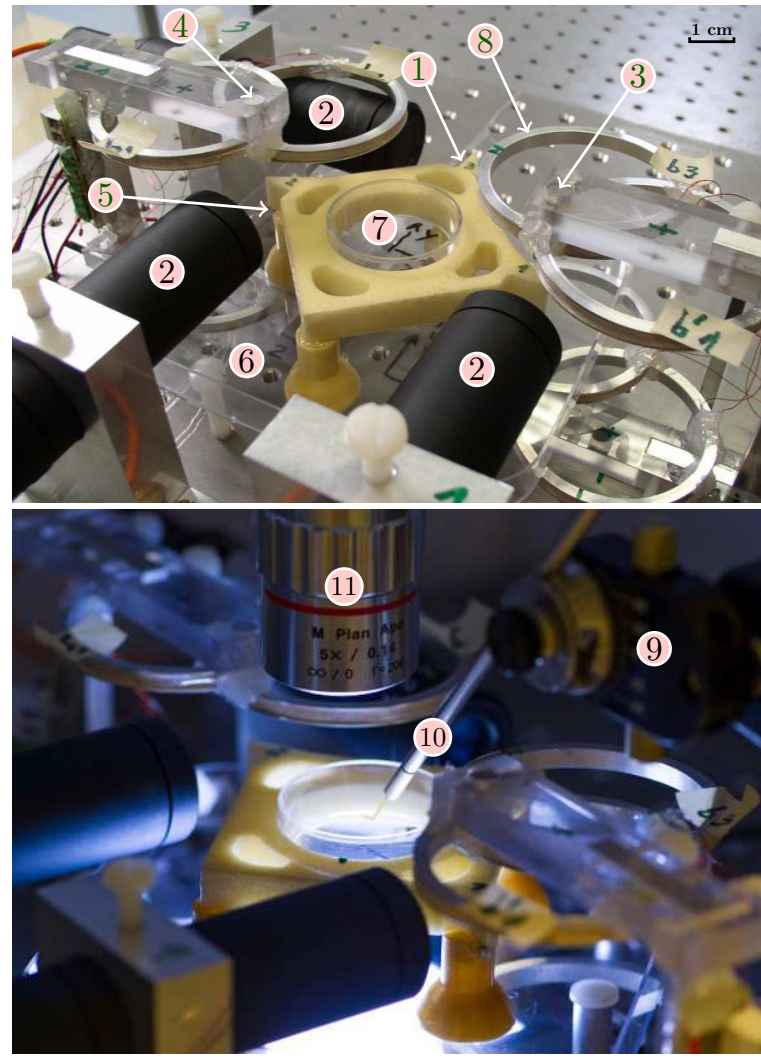

Figure 7: Experimental setup of the force sensor.
(5) platform permanent magnets $M_{1}^{f}, M_{2}^{f}$,

6) liquid tank filled with water,

(7) special Petri dish filled with a culture medium in which the oocyte is placed,

(8) coils used to calibrate the force measurement chain.

The following elements are specially added for oocytes characterisation:

(9) Eppendorf manipulator,

(10) micropipette holder,

(11) vertical microscope.

This entire device is placed on an anti-vibration table and into a closed chamber to minimize ground vibration and aerial disturbances. To ensure a constant temperature in the closed chamber, the device is always turned on at least 6 hours before any measurements. It is found that $T_{\text {chamber }}$ is equal to $29^{\circ} \mathrm{C} \pm 1^{\circ} \mathrm{C}$ with a room temperature $T_{\text {room }}$ equals to $23^{\circ} \mathrm{C} \pm 1^{\circ} \mathrm{C}$. Without the opportunity to regulate the temperature to $37^{\circ} \mathrm{C}$ and to inject $\mathrm{CO}_{2}$ gas, all the measurements are time constrained and have a duration shorter than ten minutes to avoid oocyte deterioration. Similar conditions are used by Vidberd for optical characterisation of oocytes [27]. A vertical microscope placed above the Petri dish provides a magnified view of the oocyte during its mechanical characterisation.

A sterilized $35 \mathrm{~mm}$ diameter Petri dish is used. A well of $5 \mathrm{~mm}$ in diameter is machined in the center of the Petri dish. The oocyte is placed in this well. The micropipette moved by the manipulator is positioned in order to push the oocyte against the vertical wall of the well, as shown in Fig. 1. When the oocyte is compressed by the micropipette, the resulting force $\vec{F}$ against the wall is measured by the platform. The culture medium is correctly filled in order to ensure that the meniscus formed on the micropipette and the vertical wall of the Petri dish have no mutual interaction. The remaining force interactions between the micropipette and the edges of the Petri dish are studied in Section 3. 


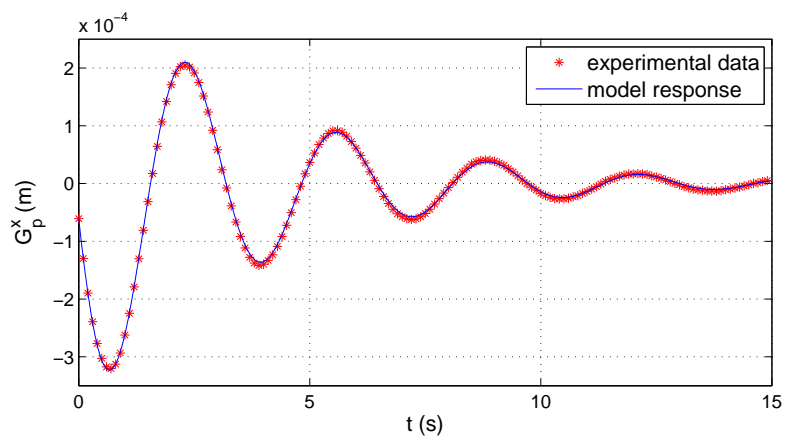

Figure 8: Comparison between the free response of the identified model and the displacement measurement of the platform.

\subsection{Calculation of the platform displacements}

The three confocal sensors $C_{1}, C_{2}$, and $C_{3}$, provide three distance measurements $l_{1}, l_{2}$, and $l_{3}$, that correspond to the distance between the sensors and the deflectors (see Fig. 6). The geometric transformation between the three $l_{i}$ distances and $G_{p}$ is [26]:

$G_{p}^{x}=\frac{\delta\left(2 \delta l_{1}-2 \delta l_{2}+4 \delta l_{3}-l_{1}{ }^{2}+l_{2}{ }^{2}\right)}{4 \delta^{2}+l_{1}{ }^{2}-2 l_{1} l_{2}+l_{2}{ }^{2}}$

$G_{p}^{y}=$

$\frac{\delta\left(l_{1}{ }^{2}-2 l_{1} l_{2}+2 l_{1} l_{3}+l_{2}{ }^{2}-2 l_{2} l_{3}+2 \delta l_{1}+2 \delta l_{2}\right)}{4 \delta^{2}+l_{1}{ }^{2}-2 l_{1} l_{2}+l_{2}{ }^{2}}$

where $\delta$ is the geometric characteristic of the platform that corresponds to the distance $M_{1}^{f} M_{2}^{f}=2 \delta$ between the magnets $M_{f}^{1}$ and $M_{f}^{2}$ (and also to the distance between the confocal sensor $C_{1}$ and $C_{2}$ axis) as shown in Fig. 6.

\subsection{Calibration of the force sensing platform}

The calibration of the force sensing platform is crucial for reliable force measurements. It consists in determining the stiffness $K_{x}$ and $K_{y}$ of the magnetic springs before any measurements on the oocyte. A second order dynamic linear model corresponding to the association of a mass, a spring and a dash-pot system is written to model the behaviour of the mass of the platform, the magnetic spring and the friction of air and liquid on the floats:

$$
\begin{array}{cc}
\mathbf{M} \ddot{\vec{X}}+\mathbf{K}_{\mathbf{f}} \dot{\vec{X}}+\mathbf{K}_{\mathbf{m}} \vec{X}=\vec{F} & \\
\mathbf{M}=\left(\begin{array}{ccc}
m & 0 & 0 \\
0 & m & 0 \\
0 & 0 & J_{z}
\end{array}\right) & \mathbf{K}_{\mathbf{f}}=\left(\begin{array}{ccc}
K_{f}^{x} & 0 & 0 \\
0 & K_{f}^{y} & 0 \\
0 & 0 & K_{f}^{\psi}
\end{array}\right)
\end{array}
$$

where $\mathbf{M}$ is a matrix including the mass $m$ and rotational inertia $J_{z}$ of the platform and $\mathbf{K}_{\mathbf{f}}$ is a matrix representing the friction. $\mathbf{K}_{\mathbf{m}}$ is the same matrix as in Eq. 1. The mass $m$ is measured with a calibrated precision balance ${ }^{6}$ with a resolution set to $0.01 \mathrm{mg}$.

A set of fixed coils have been added to the device (see Fig. 7). They are able to apply an unknown force $\vec{F}$ onto the platform and to independently control the component $F_{x}$ (or $F_{y}$ ) of $\vec{F}$. When a component $F_{x}$ (resp. $F_{y}$ ) is applied, it provides nonzero initial conditions on the position $G_{p}^{x}$ (resp. $G_{p}^{y}$ ) of the platform. The position is experimentally measured during a 15 second time interval after the cancellation of $F_{x}$ (or $\left.F_{y}\right)$. This measurement corresponds to the zero-input response of the platform $(\vec{F}=0$ in Eq. (4) with some unknown initial conditions on the position and the velocity of the platform). The Matlab Identification Toolbox ${ }^{7}$ is used to calculate the parameters of Eq. (4). The detailed procedure is the following:

- measurement outside the device of the mass $m$ of the platform, with a precision balance,

- generation of a current step into the coils to produce a force component $F_{x}\left(\operatorname{resp} . F_{y}\right)$,

- 10 seconds delay,

- cancellation of the current step $(\vec{F}=0)$,

- 2 seconds delay,

- measurement of the platform position $G_{p}^{x}$ (or $G_{p}^{y}$ ) during a time interval of 15 seconds (see Fig. 8),

\footnotetext{
${ }^{6}$ Mettler-Toledo MS105DU

${ }^{7}$ provided by Mathworks
} 


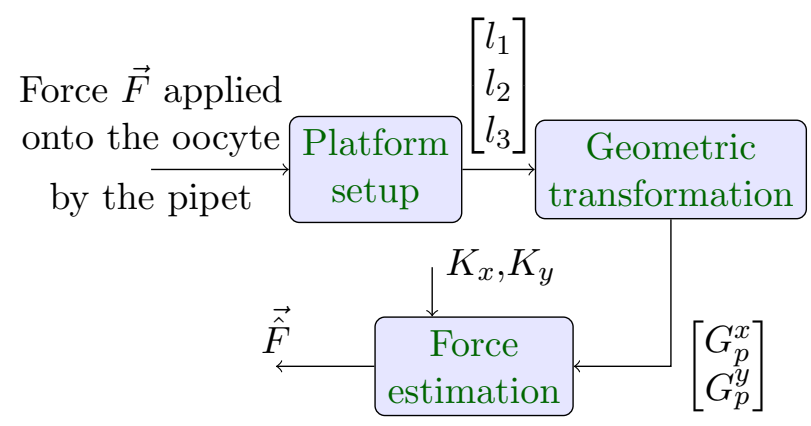

Figure 9: Measuring chain of the force sensing platform.

- identification of model parameters and initial conditions in $x$ (resp. $y$ ) direction using the Matlab model identification toolbox,

- simulation of the model for an elapsed time of 15 seconds with the identified initial conditions,

- comparison of the response time of the model to the experimental data $G_{p}^{x}$ (resp. $\left.G_{p}^{y}\right)$ measured (see Fig. 8),

- extraction of $K_{x}$ and $K_{y}$ from the model,

- activation of the force estimation on the experimental device.

The temporal response of the model given in Fig 8 shows a good accordance with the experimental data, insuring correct reliability of the force estimation. As with every micro and nanoforce sensor, it is difficult to quantify reliability of the force estimate. (No micro and nanoforce standards are available.) The difference between the data and the model is due to force disturbances that come from the room environment where the experimental device is placed. Aerial disturbances and ground vibrations, in particular, have a great influence on the calibration quality and have to be maintained as low as possible during the calibration process, as well as during force measurements. The synopsis of the measuring chain is given in Fig. 9. Typically, the resolution of the force estimation is lower than $\pm 20 \mathrm{nN}$.

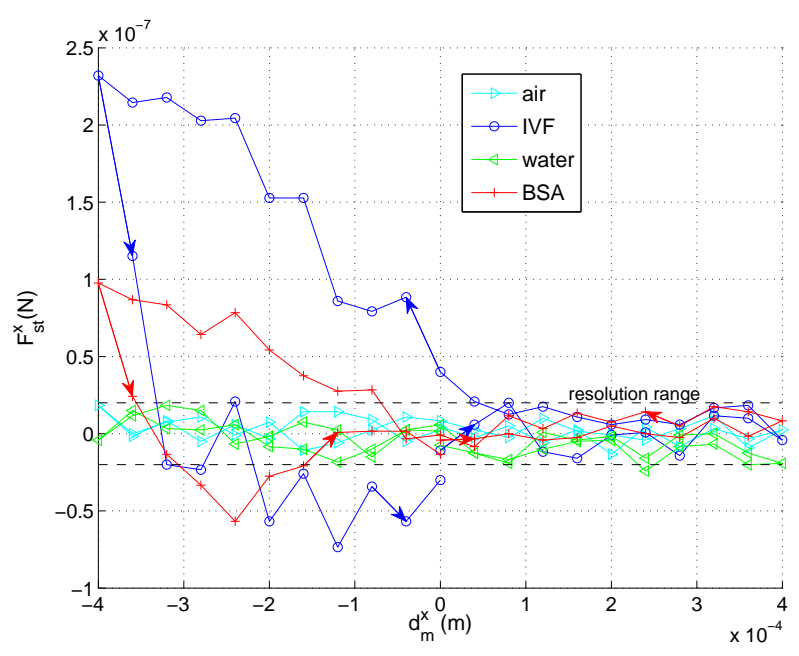

Figure 10: Measurement of surface tension disturbances for different liquid mediums at $29^{\circ} \mathrm{C}$.

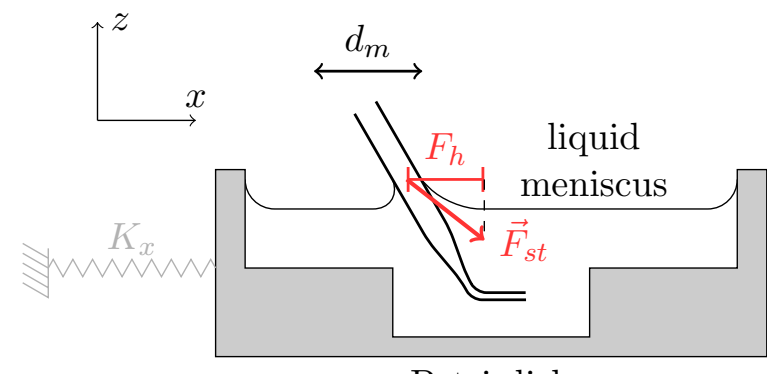

Petri dish

Figure 11: disturbance force $\vec{F}_{\text {st }}$ generated by the surface tension depending on the position $d_{m}$ of the micropipette. 


\section{Characterization of the culture medium}

Once placed in its correct position inside the IVF medium $^{8}$, the body of the micropipette goes through the surface of the liquid and a mechanical interaction is possible between them. Thus, prior to oocyte stiffness measurements, the sensor response in different liquid medium is analyzed. Each liquid medium is stored in a fridge at $4^{\circ} \mathrm{C}$ in its primary packaging. A volume sample of $1,4 \mathrm{ml} \pm 0,1 \mathrm{ml}$ is taken with a pipette and placed into the $35 \mathrm{~mm}$ diameter Petri dish inside the chamber. The measurement of the liquid medium is started ten minutes after to ensure that the temperature of the sample is equal to that of the chamber $\left(29^{\circ} \mathrm{C}\right)$. Then, the following experimental measurement protocol is used:

- positioning of the micropipette $1 \mathrm{~mm}$ above the surface of the medium in the center of the Petri dish,

- $20 \mu \mathrm{m} / \mathrm{s}$ vertical displacement of the micropipette until it reaches a position $100 \mu \mathrm{m}$ above the bottom of the Petri dish (see Fig. 11),

- successive horizontal $x$ displacements of the micropipette by steps of $40 \mu \mathrm{m}$ at $20 \mu \mathrm{m} / \mathrm{s}$ on a displacement range that extends from 0 to 400 $\mu \mathrm{m}$, then to $-400 \mu \mathrm{m}$ and followed by a return to zero,

- a time-delay of 10 seconds is performed before the force acquisition at the end of each step to insure a quasi-static configuration in order to cancel the viscous friction force.

All stop and go movements of the micropipette are performed automatically by the Eppendorf manipulator which has been programmed to follow this protocol. Fig. 10 shows the obtained results for four different mediums: air, water, the IVF medium and bovine serum albumin (BSA). The test without liquid (air medium) is taken as a reference. In this test, the oscillations of the curve represent the measurement noise and the maximum reached by these

\footnotetext{
${ }^{8} \mathrm{IVF}^{\mathrm{TM}}$ Vitrolife, Sweden
}

oscillations gives the resolution range of $\pm 20 \mathrm{nN}$ of this particular setup. The disturbances measured for a Petri dish filled with water do not show any influence of the water (same noise amplitude as in air).

The IVF medium used for oocyte characterisation is a solution composed of a bicarbonate-buffered medium containing human serum albumin and penicillin $\mathrm{G}$ as an antibacterial agent. Once placed in its correct position inside this IVF medium, a disturbance force is measured by the platform when the micropipette is moved in the horizontal direction (see Fig. 10). This force, which is different than the one obtained with air or liquid medium, can degrade the measurement of the force applied onto the oocyte. It is inferred that this force may be due to the surface tension of the IVF medium. Indeed, the sum of the surface tension at the junction of the micropipette, the air, and the liquid medium is equivalent to a disturbance force $\vec{F}_{s t}$ with a horizontal component $F_{h}$ which can be directly measured by the sensor (see Fig. 11). This force is the result of the three surface tensions between the air/liquid/solid interfaces. The albumin present in the culture medium acts as a surfactant which modifies the surface tension of the liquid [12]. Surface tensions of water and IVF medium measured by the Wilhelmy plate (Kruss tensiometer) are $\gamma_{\text {water }}=71$ $\mathrm{mN} / \mathrm{m}$ and $\gamma_{I V F}=54 \mathrm{mN} / \mathrm{m}$, respectively.

A test with a bovine serum albumin (BSA) prepared with a concentration of $10 \mathrm{~g} / \mathrm{l}$ and presenting a surface tension $\gamma_{B S A}$ equal to $\gamma_{I V F}$ shows a force profile with the same tendency as the IVF medium with a maximum disturbance force of 100 $\mathrm{nN}$ for BSA versus $230 \mathrm{nN}$ for IVF medium (see Fig. 10). This preliminary investigation shows that the albumin, which acts as a surfactant, plays a role in the measured force disturbance. This will degrade the force measurement on oocytes and there is no IVF medium free of albumin that could be used to avoid this force disturbance. Further experiments are under investigation to study the effect of the different compounds of the IVF solution on its surface tension. 


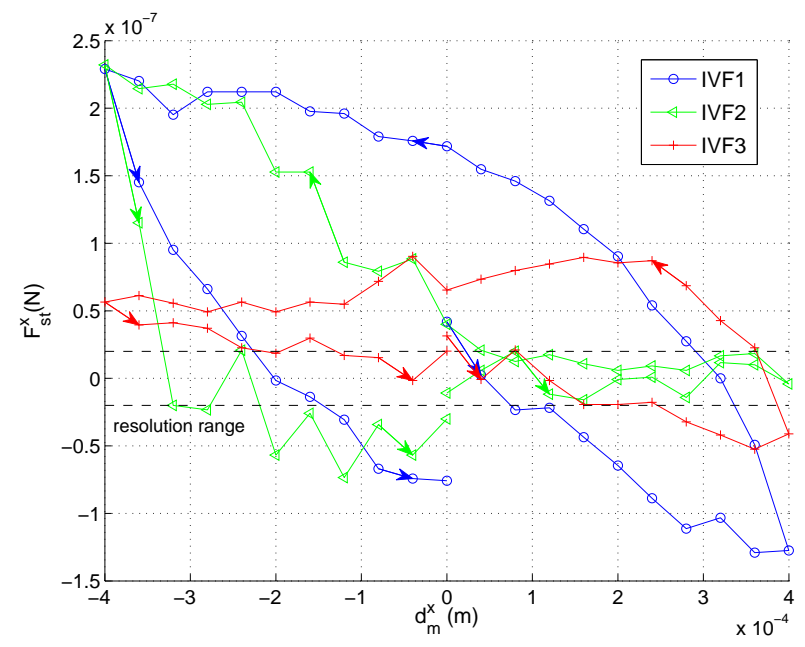

Figure 12: Measurement of surfaces tension disturbances for three different packaging syringes of IVF medium at $29^{\circ} \mathrm{C}$.

Additional investigation has been performed in order to determine the disturbance curve of the IVF medium. Measurements with culture medium coming from different packaging syringes show the same trend but the value of the disturbance force is unpredictable (see Fig. 12). A sample has been kept in the chamber at $29^{\circ} \mathrm{C}$ in order to perform measurement at different time intervals and during several hours. No significant differences have been measured during the ageing of the medium for consecutive measurements at constant temperature, on a sample coming from the same packaging.

The different responses of the sensor in water, IVF, or BSA mediums should not be due to the drag force. This force is the resistance to the motion of the micropipette and depends on the viscosity of the solution, on the shape, and on the velocity of the moving object. As the measurements are carried out in static conditions (10 seconds after each displacement of the micropipette), the drag force should be negligible. However, the effect of waiting time before the force measurements has to be studied. The physical phenomenon that provides the force hysteresis in IVF medium remains unexplained. To determine the effect of the IVF medium disturbance when the

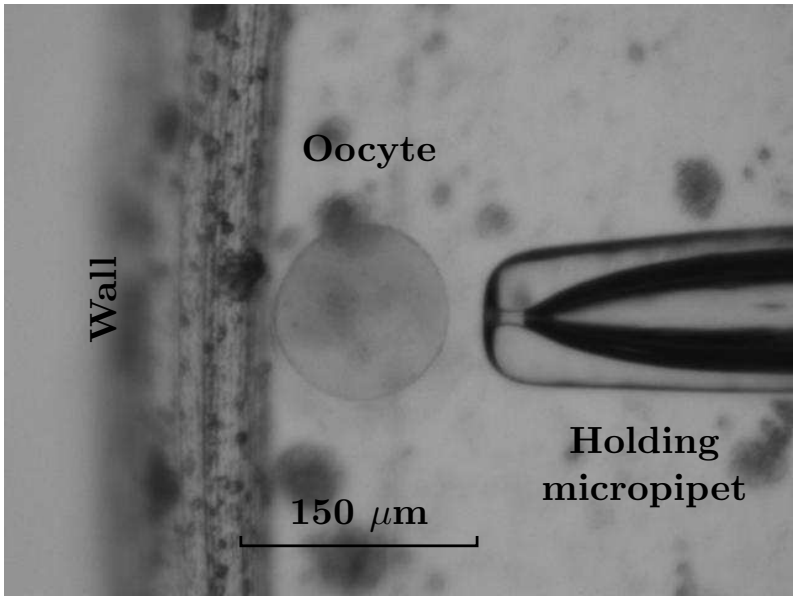

Figure 13: Oocyte correctly placed against the wall and ready for a loading cycle with an holding micropipette.

oocyte is characterized, it is proposed that the disturbance after each stiffness measurement is evaluated and compensated for, as shown in Section 4.3.

\section{Experimental measurement of oocytes stiff- ness}

\subsection{Oocytes preparation}

Oocytes used in this study are immature oocytes collected during ICSI protocol. Before the microinjection, the cumulus cells surrounding the oocyte are cleared away by association of hyaluronidase digestion for about 20 seconds (80 IU Hyaluronidase in Flushing, Fertipro, Belgium), a mechanical removal of the cumulus is performed using denudation pipettes (SWEMED by Vitrolife, Sweden) and finally, oocytes are rinsed in IVF medium and individually observed under an inverted microscope $(\times 200$ magnification). The mature oocytes at the metaphase II (MII) meiotic maturation stage, with the presence of the first polar body and the absence of germinal vesicle, are reserved for the assisted reproductive technology (ART) and the injection of a single spermatozoa into the cytoplasm of the oocyte is performed. 
The immature oocytes, at the germinal vesicle (GV) stage equal to the prophase stage of the first meiotic division, or at any stages between the germinal vesicle breakdown stage (GVBD) and the anaphase of the meiosis I, are dismissed and used for the present study. For technical reasons, the exact meiotic stage of these oocytes cannot be verified so they are classified as GVBD/MI immature oocytes. These immature oocytes are incubated in the usual IVF medium at $37^{\circ} \mathrm{C}$ and $5 \% \mathrm{CO} 2$ for a duration of 1 to 3 hours, until the stiffness measurement is performed. In these culture conditions, some of these oocytes can progress to the final maturation process and reach the MII stage. Two types of oocytes are individualized for this study, the group of the oocytes which reaches the MII stage and the group which does not reach the MII stage and that is classified as GVBD/MI. The use of these immature oocytes has been approved for our experiments by the clinical ethics committee of Besançon University Hospital on June 9th 2010.

The force sensing platform is prepared as follows: the special Petri dish is initially filled with IVF medium and placed onto the platform which has to be configured for force measurements (calibration already done). The measurement of the temperature of the IFV medium placed into the sensor chamber gives a temperature $T_{I V F}$ equals to $29^{\circ} \mathrm{C}$. After the maturation process, the oocytes are carried near the force measurement platform into an insulated box. Each oocyte is individually picked up with a Pasteur pipette and released into the machined well at $29^{\circ} \mathrm{C}$. The operator uses the Eppendorf manipulator equipped with a micropipette to push the oocyte and place it in a correct position against the well border as shown in Fig. 13. The diameter $\phi$ corresponding to the diameter of the external face of the zona pellucida is measured using the microscopic view. The diameter $\phi_{c}$ of the cytoplasm is also measured to provide the morphology of the oocyte.

\subsection{First experiment on an oocyte}

The first oocyte $O_{1}$ to be characterized is classified GVBD/MI with a diameter $\phi$ equal to $160 \mu \mathrm{m}$ $\left(\phi_{c}=112 \mu \mathrm{m}\right)$. The micropipette used is $35 \mu \mathrm{m}$ in

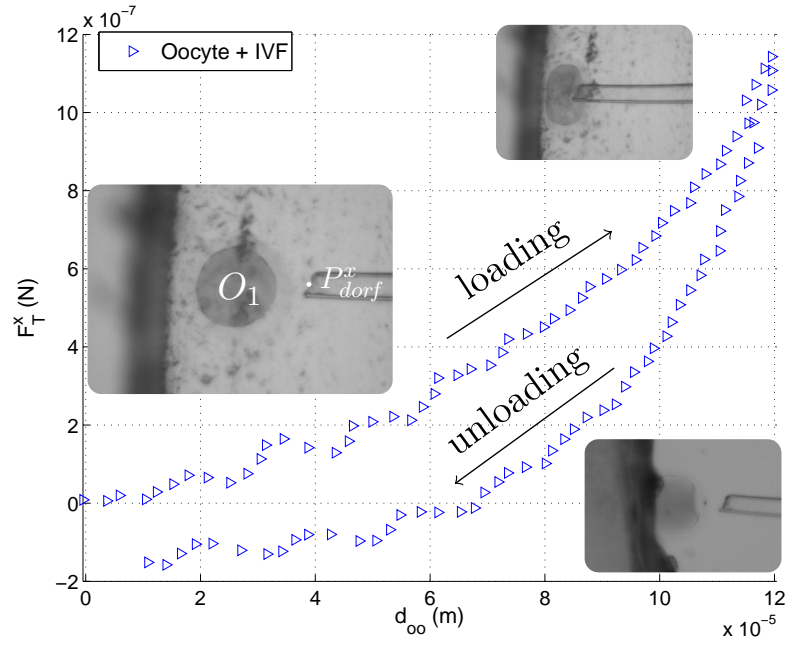

Figure 14: Oocyte $O_{1}$ stiffness characterisation without disturbance correction of the IVF medium.

diameter. Fig. 14 shows the curve obtained for a loading until the compression length of the oocyte $d_{o o}$ (see Fig. 2) reach $120 \mu \mathrm{m}$, followed by an unloading until the tip of the micropipette $\left(P_{d o r f}^{x}\right.$ coordinate) returns to its initial position. The speed of the micropipette is held constant by the internal control of the Eppendorf manipulator and is equal to $10 \mu \mathrm{m} / \mathrm{s}$ for loading and unloading cycles. The entire cycle is programmed and performed automatically. The low speed insures a quasi-static behaviour. The oocyte compression length $d_{o o}$ is calculated from the difference of the relative displacement of the Eppendorf manipulator and the platform position. The compression length $d_{o o}$ is equal to zero when the micropipette tip reaches the oocyte placed against the well border without any deformation or force measured by the sensor.

The curve $F_{\text {Oocyte }+I V F}^{x}\left(d_{o o}\right)$ given in Fig. 14 is the result obtained with the oocyte labelled $O_{1}$. The behaviour of this oocyte during loading is not linear. When the compression length $d_{o o}$ reaches $120 \mu \mathrm{m}$, the measured force is $1.15 \mu \mathrm{N}$ and represents the sum of the oocyte and the IVF medium contributions. During unloading, the hysteresis measured corresponds to the plastic deformation (see unloading curve in Fig. 14). The oocyte recovers its initial shape a few 


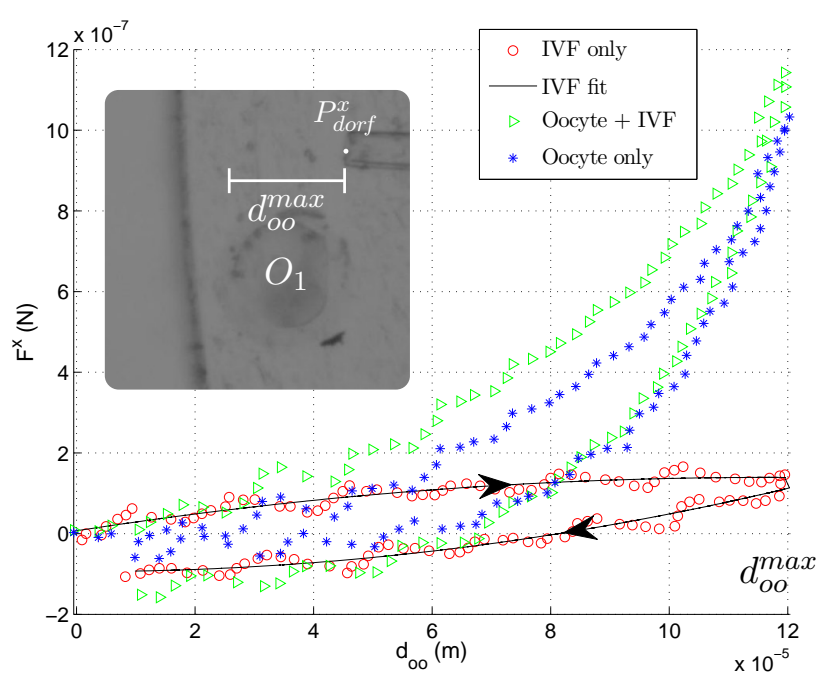

Figure 15: Oocyte $O_{1}$ stiffness characterisation including IVF medium characterisation and compensation.

minutes after the test. One can notice some residual oscillations on the curve which may be due to the poor quality of the speed control law implemented inside the Eppendorf manipulator. The disturbance contribution of the IVF medium has to be removed from this curve in order to have the single oocyte contribution.

\subsection{Compensation of the IVF medium disturbance}

After each measurement on the oocyte, a special cycle is programmed inside the Eppendorf manipulator in order to reproduce the relative displacement of the micropipette into the IVF medium alone at the same speed as during the test on the oocyte, but this time the tip is placed beside the oocyte as shown in Fig. 15. This relative displacement is limited to $d_{o o}^{\max }$, which is the maximum displacement $\left(12 \times 10^{-5} \mathrm{~m}\right.$ in Fig. 14) performed previously, during the oocyte $O_{1}$ characterisation. The curve $F_{I V F \text { only }}^{x}\left(d_{o o}\right)$ corresponding to the IVF medium alone (see Fig. 15) is obtained. A second-order polynomial $F_{I V F \text { fit }}^{x}\left(d_{o o}\right)$ is fitted to the curve $F_{I V F \text { only }}^{x}\left(d_{o o}\right)$. Finally the curve $F_{\text {Oocyte only }}^{x}\left(d_{o o}\right)$ corresponding to the oocyte alone is calculated with the following formula:

$$
F_{\text {Oocyte only }}^{x}\left(d_{o o}\right)=F_{\text {Oocyte }+I V F}^{x}-F_{I V F \text { fit }}^{x}
$$
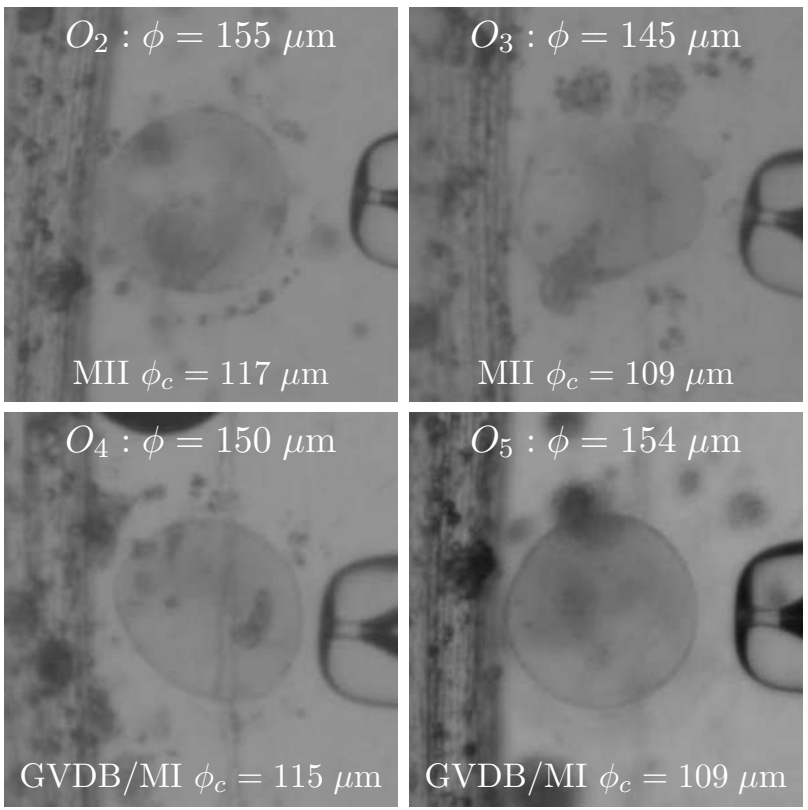

Figure 16: Visualization of the four different oocytes.

The influence of the IVF medium disturbance is not negligible. Its compensation offers a better stiffness characterisation of the oocyte. At the end of the unloading curve corresponding to "Oocyte only", one can notice that the force becomes close to zero with a negative trend just higher than the resolution of the sensor $( \pm 20 \mathrm{nN})$. This is due to an adhesion force between the micropipette tip and the zona pellucida.

All the measurements presented in the next Sections include a systematic IVF medium characterisation and compensation. Only the curves $F_{o v o}^{x}\left(d_{o o}\right)$ are given.

\subsection{Comparison between different oocytes}

Four different oocytes have been characterized in order to compare their stiffness. The micropipette used to compress the oocytes is a standard holding micropipette of $85 \mu \mathrm{m}$ in diameter which offers a large frontal plane. The oocytes are labelled $\mathrm{O}_{2}, \mathrm{O}_{3} \mathrm{O}_{4}$ and $O_{5}$. A snapshot of each one associated with its diameter and maturity stage is given in Fig. 16. The 


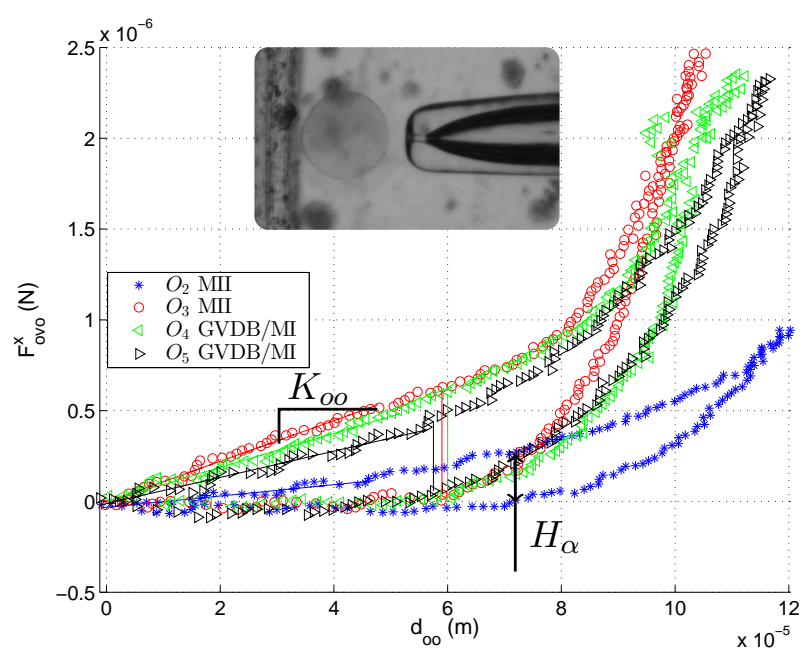

Figure 17: Stiffness comparison between several oocytes using holding micropipette.

\begin{tabular}{|c|c|c|}
\hline Label & $K_{o o}(\mathbf{m N} / \mathbf{m})$ & $H_{\alpha}(\mu \mathbf{N})$ \\
\hline \hline$O_{2}(\mathrm{MII})$ & 3.1 & 0.26 \\
$O_{3}(\mathrm{MII})$ & 11.2 & 0.57 \\
$O_{4}(\mathrm{GVBD} / \mathrm{MI})$ & 9.3 & 0.58 \\
$O_{5}(\mathrm{GVBD} / \mathrm{MI})$ & 7.2 & 0.38 \\
\hline
\end{tabular}

Table 1: Results for the four oocyte characterisations.

first two are mature (metaphase II) and the others are immature (GVBD/MI). Their stiffness characteristic curves are given in Fig. 17. Two parameters, $K_{o o}$ and $H_{\alpha}$, are defined to quantify their mechanical behaviour (see Fig. 17). The parameter $K_{o o}$ is the linear stiffness of the oocyte calculated by fitting a straight line on the loading linear response, when $d_{o o}$ is lower than $50 \mu \mathrm{N}$. The second parameter $H_{\alpha}$ is the maximum hysteresis amplitude between loading and unloading. It represents the oocyte's ability to recover its initial shape during the test: the greater $H_{\alpha}$, the greater the residual plastic deformation that the oocyte finally recovers a few minutes after the test. A low value of $H_{\alpha}$ means that the oocyte tends to an elastic behaviour without energy dissipation. Table 1 summarizes the behaviour of each oocyte. Distinction between mature and immature oocytes cannot

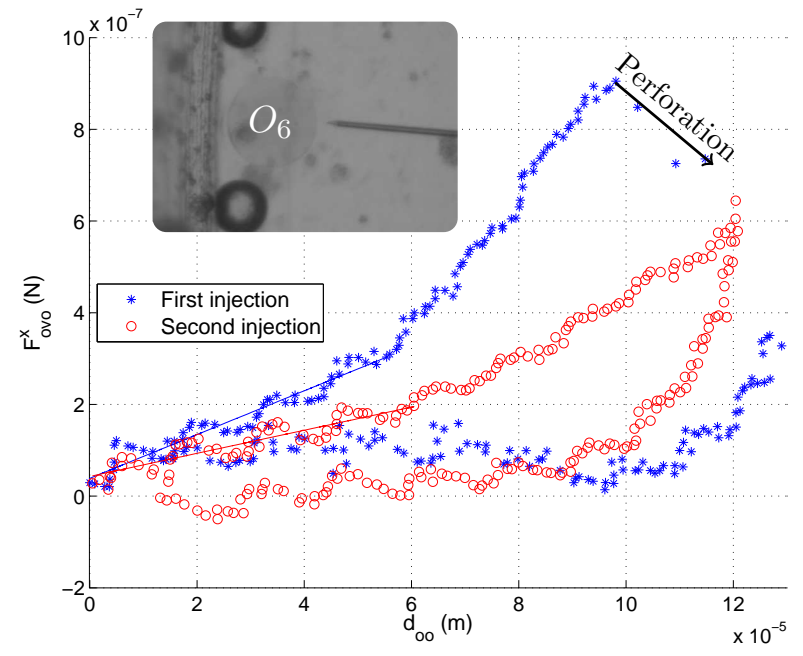

Figure 18: Force measurement for two successive injection cycles on the oocyte $\mathrm{O}_{6}$.

be made based on these mechanical criteria. A significant difference is detected between the two mature oocytes, $\mathrm{O}_{2}$ and $\mathrm{O}_{3}$. The $\mathrm{O}_{3}$ oocyte has reached its meiotic competence, but contrary to the $\mathrm{O}_{2}$, it has not reached its optimal terminal diameter. An interpretation of this result could be that the higher stiffness of $O_{3}$ may indicate immaturity of the cytoplasm. The stiffness of $\mathrm{O}_{2}$ is lower than $\mathrm{O}_{3}$, but it has a greater capacity to recover its shape during the unloading.

\subsection{Tests with an injection needle}

Fig. 18 shows the results obtained for an injection cycle done on the metaphase II oocyte $O_{6}$ with a diameter of $150 \mu \mathrm{m}\left(\phi_{c}=108 \mu \mathrm{m}\right)$. An empty (without sperm) injection needle with a diameter of $6 \mu \mathrm{m}$ is used. On the first injection cycle, the perforation of the cytoplasmic membrane occurs for $d_{o o}=98 \mu \mathrm{m}$ when the force applied to the oocyte reaches $F_{\text {ovo }}^{x}=0.90 \mu \mathrm{N}$. The stiffness measured is $K_{o o}^{1}=4.8 \mathrm{mN} / \mathrm{m}$. No sperm is expelled into the oocyte and the micropipette is simply removed from the oocyte. After a delay of 15 minutes, a second injection cycle is done on the same oocyte. This time, no perforation occurs and the stiffness measured decreases: $K_{o o}^{2}=2.8 \mathrm{mN} / \mathrm{m}$. This phenomenon could 


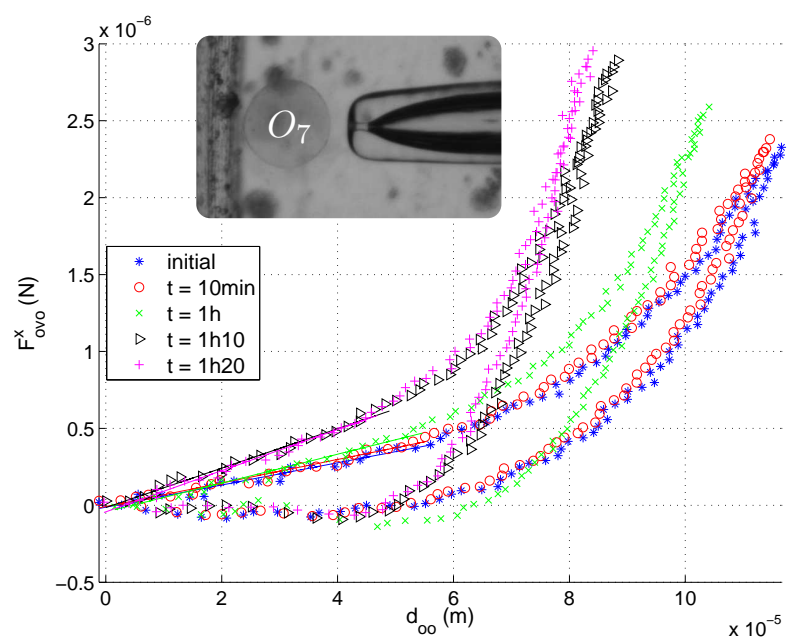

Figure 19: Evolution of the stiffness of the dying oocyte $O_{7}$ (GVBD/MI)

be caused by the stress produced by the initial perforation.

\subsection{Evolution of the characteristics of an oocyte placed in bad culture conditions}

Fig. 19 shows the results obtained for consecutive loading cycles on the GVBD/MI oocyte $O_{7}$ with a diameter of $154 \mu \mathrm{m}\left(\phi_{c}=111 \mu \mathrm{m}\right)$. The test is performed with a standard holding micropipette with a diameter of $85 \mu \mathrm{m} .10$ minutes after an initial loadunload cycle, a second identical cycle is performed. Then, new cycles are performed 1 hour later, $1 \mathrm{~h} 10$ later and $1 \mathrm{~h} 20$ later. The oocyte stays at chamber temperature in the same Petri dish and in the same IVF medium during the entire duration of this test. In these culture conditions, the normal maturation process is perturbed. Nevertheless, this test can provide interesting information on the oocyte evolution once placed in non-optimal conditions. It remains to establish that a single oocyte showing a similar mechanical evolution during its normal maturation could be considered evolving incorrectly. Table 2 shows the evolution versus time of the two parameters $K_{o o}$ and $H_{\alpha}$ corresponding to the oocyte $O_{7}$. An important increase of the oocyte stiffness is visible, showing that a mechanical variation of the oocyte

\begin{tabular}{|l|c|c|}
\hline Time & $K_{\text {oo }}(\mathbf{m N} / \mathbf{m})$ & $H_{\alpha}(\mu \mathbf{N})$ \\
\hline \hline initial & 7.2 & 0.45 \\
$\mathrm{t}=10 \mathrm{~min}$ & 7.6 & 0.43 \\
$\mathrm{t}=1 \mathrm{~h}$ & 9.3 & 0.68 \\
$\mathrm{t}=1 \mathrm{~h} 10$ & 12.8 & 0.69 \\
$\mathrm{t}=1 \mathrm{~h} 20$ & 13.4 & 0.67 \\
\hline
\end{tabular}

Table 2: Results for the consecutive loadings on the oocyte $O_{7}$ (GVBD/MI).

structure can be observed using repeated load-unload cycles. This increase is greater after a delay of one hour and is confirmed by the evolution of the $H_{\alpha}$ parameter. Nevertheless, the question of the neutrality of these measurement cycles on the oocyte evolution (mechanical stress or fatigue), still remains to be investigated.

\section{Conclusion and perspectives}

The human oocyte selection plays a critical role in ART success rate. In order to improve this rate, new instrumentation is necessary to extend the usual protocols used by physicians to determine the oocytes' quality and maturity level. This new instrumentation must be compatible with ART constraints.

Among the new technologies available to determine the maturity level of a human oocyte, the characterisation of its mechanical properties is potentially interesting and has to be investigated. This study requires a force sensor with a very low stiffness to have a high resolution and also a large measurement range if large deformations have to be characterised. Force sensors based on magnetic springs have such characteristics and could potentially be a part of this new instrumentation.

This article presents some preliminary results obtained with a floating platform using a magnetic spring dynamometer. Since the final objective is to sort human oocytes and since it is difficult to extrapolate data obtained with an animal model 
to human ART, these preliminary results are only carried out with human oocytes. The preliminary results demonstrate that is it possible to visualize mechanical load-unload cycles applied on human oocytes despite their very low stiffness (around 10 $\mathrm{mN} / \mathrm{m}$ ). The human oocyte elastic behaviour during the mechanical load (compression stage) seems linear for low loading and non-linear for high loading. After a high loading, a plastic behaviour is associated with the unload stage. The "force-compression length" curves obtained are different for different oocytes and seem to evolve with time. Two basic parameters have been suggested to quantify the oocytes' mechanical behaviour, but the question of extraction of discriminant parameters from these curves or from the raw force signal remains open and has to be investigated. The evolution of these parameters could be correlated to the global cytoplasmic and nuclear maturity of the investigated oocytes

The main drawback of the actual device used to perform these cycles of measurement is that the complex mechanical influence of the IVF medium is not negligible and has to be compensated for, which makes the experimental measurement protocol rather complex. A special Petri dish also has to be used. The development of a new force-sensing platform design, based on magnetic springs that avoid these drawbacks, is the next step to perform. To be effective, this new technology should be fully compatible with physician's protocols and constraints, but should also provide analysis methods, preferably on a multimodal basis, to improve the reliability of oocyte maturity diagnosis. Both mechanical and optical approaches are thought effective to establish a reliable diagnosis despite the variability of the oocytes' evolution $[28,29]$. The mechanical tests will provide the stiffness determination of oocytes for different loads. Referenced optical characterisation techniques based on Karhunen-Loeve tranform [30] or a spectral absorption analysis [31] are also promising because they have no deleterious effects on oocytes. Thus, a potentially interesting future design should be based on a modular micromechanical / optical device including a high sensitive nanoforce sensor based on magnetic springs. All parts in direct contact with the oocyte have to be low-cost, sterile and disposable.

The oocyte state at a given time can be represented by a feature vector extracted from all available multiphysics data (mechanical, optical, morphological, morphometrics) and each oocyte maturity process is then represented by a specific trajectory in the multi-dimensional parametric space associated to the feature vector components. With such representation, the maturity diagnosis could be done thanks to supervised classification approaches [32]. These approaches are based on a training process for which the feature vectors of some specific oocytes are labelled in time according to the different stages of maturity (set-membership approaches). After this training process, the classification algorithm goal is to provide a label affectation for each new feature vector evolving in time. In the specific case of the IFV context, the choice of the feature vector components is critical for the effectiveness of the classification and the classification algorithm should also be able to manage the unavoidable uncertainty that is associated with each labeling during the training process. Classification algorithms based on Dempster-Shafer theory of evidence that enable such framework could provide interesting methods for investigation [33].

\section{Acknowledgement}

This collaborative work has been supported by the Labex ACTION project (contract "ANR-11-LABX01-01")

\section{Bibliography}

[1] Y. Murayama, C.E. Constantinou, and S. Omata. Micro-mechanical sensing platform for the characterization of the elastic properties of the ovum via uniaxial measurement. $J$. Biomech., 37:67-72, 2004.

[2] T. Woodruff and L. Shea. A new hypothesis regarding ovarian follicle development: ovarian rigidity as a regulator of selection and health. Journal of Assisted Reproduction and Genetics, 28(1):3-6, 2011. 
[3] K. Kim, X. Liu, Y. Zhang, J. Cheng, X. Yu Wu, and Y. Sun. Manipulation at the nanonewton level: Micrograpsing for mechanical characterization of biomaterials. In IEEE International Conference on Robotics and Automation, pages 902-907, 2009.

[4] D. Nishi, T. Arai, K. Inoue, and T. Takubo. Measurement of the mechanical properties of living cell using micro hand and developed afm system. International conference on intelligent robots and systems, pages 1184-1188, 2005.

[5] P. Rougeot, S. Régnier, and N. Chaillet. Forces analysis for micro-manipulation. In Proc. of the IEEE International Symposium on Computational Intelligence in Robotics and Automation, espoo, Finland, pages 105-110, june 2005.

[6] F. Beyeler, S. Muntwyler, and B. J. Nelson. A six-axis mems force-torque sensor with micro-newton and nano-newtonmeter resolution. Journal of Microelectromechanical Systems, 18(2):433-441, 2009.

[7] F. Wottawah, S. Schinkinger, B. Lincoln, R. Ananthakrishnan, M. Romeyke, J. Guck, and J. K'as. Optical rheology of biological cells. Phys. Rev. Lett., 94:098103, Mar 2005.

[8] B. R. Daniels, B. C. Masi, and D. Wirtz. Probing single-cell micromechanics in vivo: The microrheology of c. elegans developing embryos. Biophysical Journal, 90(12):4712-4719, 2006.

[9] J. H. He, W. Xu, and L. Zhu. Analytical model for extracting mechanical properties of a single cell in a tapered micropipette. Applied Physics Letters, 90(2):023901-023901-3, 2007.

[10] N. Desprat, A. Guiroy, and A. Asnacios. Microplates-based rheometer for a single living cell. Review of Scientific Instruments, 77(5):055111-055111-9, 2006.

[11] G. Pesce, L. Selvaggi, A. Caporali, A. C. De Luca, A. Puppo, G. Rusciano, and A. Sasso. Mechanical changes of living oocytes at maturation investigated by multiple particle tracking. $A p$ plied Physics Letters, 95(9):093702, 2009.

[12] L. Rienzi, G. Vajta, and F. Ubaldi. Predictive value of oocyte morphology in human ivf: a systematic review of the literature. Human Reproduction Update, 17(1):34-45, 2011.

[13] Alpha scientists in reproductive medicine and ESHRE special interest group of embryology. The istanbul consensus workshop on embryo assessment: proceedings of an expert meeting. $\mathrm{Hu}$ man Reproduction, 26(6):1270-1283, 2011.

[14] M. Montag, T. Schimming, M. Köster, C. Zhou, C. Dorn, B. Rösing, H. van der Ven, and K. van der Ven. Oocyte zona birefringence intensity is associated with embryonic implantation potential in icsi cycles. Reproductive BioMedicine Online, 16(2):239-244, 2008.

[15] T. Ebner, B. Balaban, M. Moser, O. Shebl, B. Urman, B. Ata, and G. Tews. Automatic user-independent zona pellucida imaging at the oocyte stage allows for the prediction of preimplantation development. Fertil. Steril., 94(3):913-920, 2010.

[16] S. Nakamura and Y. Hiramoto. Mechanical properties of the cell surface in starfish eggs. Develop. Growth and Differ, 20(4):317-327, 1978.

[17] Y. Sun, K.-T. Wan, K.P. Roberts, J.C. Bischof, and B.J. Nelson. Mechanical property characterization of mouse zona pellucida. NanoBioscience, IEEE Transactions on, 2(4):279-286, dec. 2003.

[18] Y. Murayama, J. Mizuno, H. Kamakura, Y. Fueta, H. Nakamura, K. Akaishi, K. Anzai, A. Watanabe, H. Inui, and S. Omata. Mouse zona pellucida dynamically changes its elasticity during oocyte maturation, fertilization and early embryo development. Human Cell, 19(4):119 $125,2006$.

[19] X. Liu, R. Fernandes, A. Jurisicova, R. F. Casper, and Y. Sun. In situ mechanical characterization of mouse oocytes using a cell holding device. Lab Chip, 10:2154-2161, 2010. 
[20] Y. Murayama, M. Yoshida, J. Mizuno, H. Nakamura, S. Inoue, Y. Watanabe, K. Akaishi, H. Inui, C.E. Constantinou, and S. Omata. Elasticity measurement of zona pellucida using a micro tactile sensor to evaluate embryo quality. Journal of Mammalian Ova Research, 25(1):816, April 2008.

[21] B. Wacogne, C. Pieralli, C. Roux, and T. Gharbi. Measuring the mechanical behaviour of human oocytes with a very simple su- 8 microtool. Biomedical Microdevices, 10(3):411-419, 2008.

[22] X. Liu, J. Shi, Z. Zong, K. Wan, and Y. Sun. Elastic and viscoelastic characterization of mouse oocytes using micropipette indentation. Annals of Biomedical Engineering, [Epub ahead of print], 2012.

[23] M. Boukallel, E. Piat, and J. Abadie. Passive diamagnetic levitation : theoretical foundations and application to the design of a micro-nano force sensor. In Proc. of the IEEE/RSJ International Conference on Intelligent Robots and Systems, IROS'2003, pages 1062-1067, 2003.

[24] J. Abadie, E. Piat, S. Oster, and M. Boukallel. Modeling and experimentation of a passive low frequency nanoforce sensor based on diamagnetic levitation. Sensors and Actuators A: Physical, 173(1):227-237, 2012.

[25] A. Cherry, J. Abadie, and E. Piat. Microforce sensor for microbiological applications based on a floating-magnetic principle. In IEEE International Conference on Robotics and Automation, pages 1504-1509, 2007.

[26] A. Cherry, J. Abadie, and E. Piat. Analysis of a passive microforce sensor based on magnetic springs and upthrust buoyancy. Sensor and Actuator A: Physical, 169(1):27-36, 2011.

[27] F. Vidberg, R. Zeggari, C. Pieralli, C. Amiot, C. Roux, and B. Wacogne. Measurement of oocyte temporal maturation process by means of a simple optical micro-system. Sensors and Actuators B: Chemical, 157(1):19 - 25, 2011.
[28] R. Zeggari, B. Wacogne, C. Pieralli, C. Roux, and T. Gharbi. A full micro-fluidic system for single oocyte manipulation including an optical sensor for cell maturity estimation and fertilisation indication. Sensors and Actuators B: Chemical, 125(2):664-671, 2007.

[29] B. Wacogne, I. Ivascu, C. Pieralli, C. Amiot, L. Pazart, and C. Roux. Microsensors and image processing for single oocyte qualification: towards multiparametric determination of the best moment for fertilization. In $L P H Y$ '12 Proceedings, Calgary, Canada, 2012.

[30] C. Pieralli, B. Wacogne, C. André, C. Roux, C. Joanne, and L. Pazart. Biological qualification of oocyte maturity with the use of the karhunen-loeve transform: Computer-aided decision for selecting best oocytes before fertilization. In MEDICAL IMAGE ANALYSIS AND DESCRIPTION FOR DIAGNOSIS SYSTEMS, pages 77-84, Porto, Portugal, 2009.

[31] J. André, C. Amiot, F. Vidberg, C. Pieralli, B. Wacogne, and C. Roux. Measuring the oocyte cytoplasmic maturation process with a white light optical microsystem. Journal of Reproductive Medicine and Endocrinology, 7(4):284, 2010.

[32] C. M. Bishop. Pattern recognition and machine learning, volume 1. springer New York, 2006.

[33] E. Ramasso, M. Rombaut, and N. Zerhouni. Joint prediction of observations and states in time-series: a partially supervised prognostics approach based on belief functions and $\mathrm{knn}$. IEEE Transactions on Systems, Man, and Cybernetics, Part B: Cybernetics, 43(1):37-50, February 2013. 


\section{List of Figures}

1 Forces applied on the force sensing device when an oocyte is under a compression test. . . . . 3

2 The compressed oocyte with the forces applied on it and the resulting compression length $d_{o o}$. 3

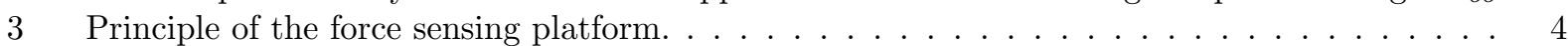

4 Behaviour of the floating platform when a force $\vec{F}$ is applied on it. . . . . . . . . . . 4

$5 \quad$ Force field generated perpendicularly to the vertical line (bt) by magnets $M_{1}^{t}$ and $M_{1}^{b}$ and

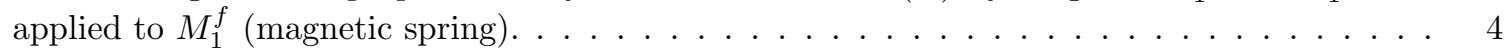

6 Displacement measurement of the platform with confocal sensors. . . . . . . . . . . . . 5

7 Experimental setup of the force sensor. . . . . . . . . . . . . . . . . . 6

8 Comparison between the free response of the identified model and the displacement measure-

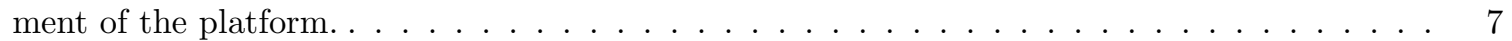

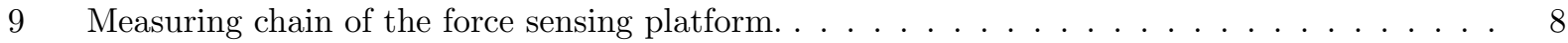

10 Measurement of surface tension disturbances for different liquid mediums at $29^{\circ} \mathrm{C} \ldots \ldots$

11 disturbance force $\vec{F}_{s t}$ generated by the surface tension depending on the position $d_{m}$ of the

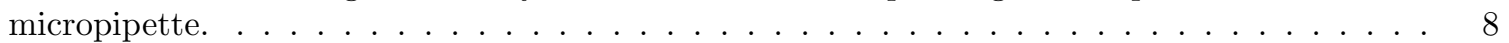

12 Measurement of surfaces tension disturbances for three different packaging syringes of IVF medium at $29^{\circ} \mathrm{C} \ldots \ldots \ldots \ldots \ldots \ldots \ldots \ldots$

13 Oocyte correctly placed against the wall and ready for a loading cycle with an holding micropipette. . . . . . . . . . . . . . . . . . . . . . 10

14 Oocyte $O_{1}$ stiffness characterisation without disturbance correction of the IVF medium. . . . 11

15 Oocyte $O_{1}$ stiffness characterisation including IVF medium characterisation and compensation. 12

16 Visualization of the four different oocytes. . . . . . . . . . . . . . . . . . . 12

17 Stiffness comparison between several oocytes using holding micropipette. . . . . . . . . . . 13

18 Force measurement for two successive injection cycles on the oocyte $O_{6} \ldots \ldots \ldots$

19 Evolution of the stiffness of the dying oocyte $O_{7}(\mathrm{GVBD} / \mathrm{MI}) \ldots \ldots \ldots \ldots$ 